\title{
Evaluation of the Relationship Between C-reactive Protein, Lactate, Procalcitonin and Albumin Levels and Procalcitonin/Albumin Ratio with SOFA and APACHE-II Scores in Emergency ICU Patients
}

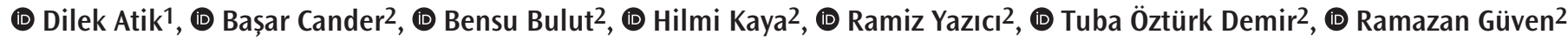 \\ 1Department of Emergency Medicine, Yozgat Bozok University, Yozgat, Turkey \\ ${ }^{2}$ Clinic of Emergency Medicine, University of Health Sciences Turkey, Kanuni Sultan Süleyman Training and Research Hospital, İstanbul, Turkey
}

\begin{abstract}
Aim: This study aimed to investigate the efficacy of C-reactive protein (CRP), lactate, procalcitonin and albumin levels and procalcitonin/ albumin ratio used as indicators of infection on mortality in critical patients admitted to the intensive care unit and their relationships with APACHE-II and SOFA scores.

Materials and Methods: This study was conducted using patients' hospital records and patient file scans. Demographic characteristics; procalcitonin, CRP, albumin and lactate levels and APACHE-II and SOFA scores were recorded. Spearman's rank correlation was used to assess non-parametric data. ROC curve analysis was performed to determine the threshold values of blood parameters. Results: A total of 61 patients were enrolled in the present study [35 males (57.4\%) and 26 females (42.6\%); average age, 69.0 years]. A positive, weakly significant association was detected between APACHE-II score and procalcitonin levels. When the APACHE-II score and lactate level and procalcitonin/ albumin ratio were evaluated,

Results: APACHE-II score was positively significant and weakly correlated with lactate and the procalcitonin/albumin ratio. A moderate negative correlation was found between albumin level and the APACHE-II score. The SOFA score was positively associated with both procalcitonin and lactate levels. SOFA score was positively significant and weakly correlated with the procalcitonin/albumin ratio.

Conclusion: Procalcitonin, lactate and albumin levels and the procalcitonin/albumin ratio can be considered prognostic markers according to the cut-off points in terms of mortality in critically ill patients. In addition, these blood parameters were found to be useful in clinical follow-up as they are related to the APACHE-II and SOFA scoring systems used in intensive care units.
\end{abstract}

Keywords: Critical patient, SOFA, APACHE-II, procalcitonin, procalcitonin/albumin ratio, lactate, CRP

\section{Introduction}

Critical patient is a term expressing the patients with higher rates of morbidity and mortality rates who need developed monitoring and treatment due to one or multiple organ or system failure $(1,2)$. Monitoring of critical care patients; intensive care units equipped with advanced technology, vital indicators are monitored and patient follow-up and treatment are provided 24 hours a day. In 1999, "Society of Critical Care Medicine" prepared admission, triage and discharge guidelines for intensive care. Intensive care admission decision; priority is divided into three according to diagnostic and objective parameter models (3).
Different scoring systems were developed for early detection and treatment planning of these patients because of higher morbidity and mortality rates. Acute Physiology And Chronic Health Evaluation II (APACHE-II) and the sequential organ failure assessment score (SOFA) are two of the aforesaid scoring systems. APACHE-II and SOFA are complementary scores. APACHE-II score, which was previously evaluated during resuscitation in emergency department, has been shown to be strongly associated with mortality (4). The SOFA score is a scoring system that effectively demonstrates treatment efficacy and course of disease (5).

Cite this article as: Atik D, Cander B, Bulut B, Kaya H, Yazııı R, Öztürk Demir T, Güven R. Evaluation of the Relationship Between C-reactive Protein, Lactate, Procalcitonin and Albumin Levels and Procalcitonin/Albumin Ratio with SOFA and APACHE-II Scores in Emergency ICU Patients. Eurasian J Emerg Med. 2020;19(2):98-104 
In the studies conducted, it was reported that laboratory parameters correlated with scoring systems $(6,7)$. C-reactive protein (CRP), procalcitonin and lactate are laboratory parameters which significantly increase in infection or inflammation. Some proteins called acute phase reactants are synthesized from the liver during infection, trauma, inflammatory events and some malignant diseases. These acute phase reactants prevent progression of the tissue damage, activate repair mechanism and try to isolate or injure the organism which causes infection (8). CRP is one of the acute phase reactants. It exists at very small quantities in serum of the healthy individuals without any change during the day (9). Serum level of the protein starts to elevate after 3 to 6 hours following onset of the inflammation, and it reaches to the highest level after 36 to 60 hours (9). Procalcitonin is a precursor of calcitonin which is secreted by $C$ cells of the thyroid gland (10). Serum level of procalcitonin is very low under normal conditions: calcitonin increases as a response to hypercalcemia whereas procalcitonin is not affected (10). Although calcitonin secretion from some organs such as liver, pancreas and lungs is known, the mechanism is not clarified yet (11). Blood level of lactate which is one of the common indicators for evaluation of liquid and hemodynamic resuscitation is found associated with mortality (12). Albumin is a naturally occurring plasma protein (13). Albumin is a negative acute phase protein. In the presence of any inflammation in the body, albumin decreases with the triggering of cytokines such as TNFoc, interleukin-1 and interleukin-6 (14). A change is detected in the levels of inflammatory indicators in serum such as CRP, lactat, albumin and procalcitonin which are widely used to show the mortality during the inflammatory process induced in critical patients. Roles of CRP, procalcitonin and lactate parameters on patient management may be explained by reflection of the severity of the inflammatory process appeared in critical patients. The purpose of this study was to investigate the efficacy of procalcitonin, CRP, lactate, albumin and procalcitonin/albumin parameters used as an indicator of infection on mortality in critical patients admitted to the Intensive Care Unit and their relationship with APACHE-II, SOFA scores.

\section{Materials and Methods}

This study was conducted retrospectively in Intensive Care Unit in Emergency Medicine Clinic of Kanuni Sultan Suleyman Training and Research Hospital of Istanbul Health Sciences University between 28.11.2018-28.03.2019. It was approved by the Ethics Committee of the Institution with the protocol number $2019 / 07 / 175$. The study was carried out from hospital records of patients and scans of patient files. Patients in the emergency intensive care unit are accepted according to the objective parameter model according to the intensive care guideline
(3). The study was performed from the hospital records of the patients and the screening of the patient files.

Procalcitonin, CRP, lactate, albumin blood parameters and APACHE-II and SOFA scores were recorded at admission to the first intensive care unit. A total of 61 patients were included in the study. Age, gender, hospitalisation diagnoses and clinical results of the patients were recorded from the patient files. Gloskow Coma scale of the patients were recorded and divided into five groups. APACHE-II Score, scoring $<10$ group 1 , scoring 11-20 group 2, scoring 21-30 group 3, and scoring $>31$ group 4 were divided into four groups. SOFA score was divided into three groups. Patients under 18 years of age, patients with cancer of etiology, patients with primary blood cell malignancy such as leukemia and lymphoma, and patients with bone marrow metastasis were excluded from the study.

\section{Statistical Analysis}

All variables were tested for normal distribution, Kolmogorov Smirnov test and parametric test criteria. The data obtained from our study were evaluated with SPSS 22.0 program. Spearman's rank correlation was used for non-parametric data. For correlation between scoring and parameters, simple correlation index was used for parameters with weak correlation. Blood parameters of procalcitonin, lactate, crp, albumin and procalcitonin / albumin levels were determined by ROC curve analysis to determine the threshold value in predicting mortality. Significance was evaluated at $p<0.05$.

\section{Scores Used:}

APACHE-II score: Developed in 1985 by Knaus et al. (15) APACHEII, the simplified version of the APACHE scoring system, is the sum of three basic scores: age, chronic health status, and one Glasgow Coma score. The APACHE-II score, which uses the worst values in the first 24 hours, is used to calculate a mortality expectancy by assessing 34 separate hospitalisations. The highest APACHE-II score the patient could receive was 71 .

SOFA score: The SOFA score is mainly used to facilitate identification of patients at risk for mortality in sepsis. It is evaluated by giving a score of 1 to 4 out of 6 parameters evaluating respiratory, cardiovascular, central nervous system, renal, coagulation and liver.

\section{Results}

Sixty-one patients were enrolled into the present study. The participants included 35 males (57.4\%) and 26 females (42.6\%) with an age average of 69.0 (min:19 max: 91). Eighty-eight point five percent $(n=54)$ of the patients had additional disease. The most common conditions reported in the patient history were 
hypertension by $41 \%(n=25)$, Diabetes mellitus by $31.1 \%(n=19)$, COPD by $29.5 \%(n=18)$ and respectively. The most common diagnoses for admission to intensive care unit were pneumonia by $54.7 \%(n=28)$, hypoxic respiratory failure $26.2 \%(n=16)$, acute renal failure by $19.7 \%(n=12)$ and sepsis by $14.8 \%(n=9)$ (Table 1). Mean hospitalisation period in the intensive care unit was 6.7 days. Evaluation of patient outcomes revealed referral to another centre by emergency ambulance by $32.8 \%(n=20)$, transfer to the clinic by $14.8 \%(n=9)$, discharge with a healthy condition by $27.9 \%(n=17)$ and exitus by $24.6 \%(n=15)$. When the patients were evaluated according to GCS, the GCS 15 was $42.6 \%(n=26)$. The GCS was $26.2 \%$ ( $n=16$ ), which was $13-14$, and the GCS $10-12$ was $18 \%$ $(n=11)$. When evaluated according to APACHE-II score, $<10$ below $4.9 \%(n=3)$, between $11-20$ and $42.6 \%(n=26)$, scores between 21 30 and $31.1 \%(n=19),>31$ and $21.3 \%(n=13)$. When the patients were evaluated according to SOFA score; Those with a score of 0 were $1.6 \%(n=1)$, those with a score of $1-4$ were $63.9 \%(n=39)$, and those with $>5$ points were $34.4 \%(n=21)$. A correlation analysis was performed to detect whether a significant correlation exists between CRP, procalcitonin, albumin, lactate and procalcitonin/ albumin among laboratory parameters and APACHE-II and SOFA (Table 2,3). A positive and weakly significant association was detected between APACHE-II and procalcitonin ( $r=0.254$ and $p=0.048$ ) (Table 2). When the APACHE-II score and lactate and procalcitonin/albumin ratio were evaluated; APACHE-II was positively significant and had a weak correlation with lactate and procalcitonin/albumin ratio $(r=0.316$ and $p=0.013, r=0.288$ and $p=0.025)$ (Table 2,3).

Asimple correlation indexwas used between the groups of APACHEII and SOFA scores and procalcitonin, lactate, procalcitonin/ albumin, which are blood parameters. Procalcitonin averages of the groups according to APACHE-II score groups were 100, 112.5, 137.5 and 157 respectively. Procalcitonin/albumin averages of the groups according to APACHE-II score groups were 100, 119.2, 148.4 and 172.9 , respectively. The correlation index of the lactate average of the groups according to APACHE-II score groups was 100, 167, 184.5 and 233 respectively (Figure 1). Procalcitonin averages of the groups according to SOFA score groups were 100, 118.5 and 160.2 respectively. Procalcitonin/albumin averages of the groups according to SOFA score groups were 100, 118.9 and 168.3, respectively. The correlation index of the lactate average of the groups according to SOFA score groups was 100, 129.7 and 178.5 respectively (Figure 2 ).

A moderate negative correlation was found between albumin and APACHE-II score $(r=-0.469$ and $p=0.000)$ (Table 2). The SOFA score was positively associated with procalcitonin $(r=0.331$ and $p=0.009$ ) (Table 2). The SOFA score was positively associated lactate $(r=0.353$ and $p=0.005)$ (Table 3 ). SOFA was positively significant and weakly correlated with Procalcitonin/albumin ratio ( $r=0.362$ and $p=0.004)$. SOFA was negatively correlated with albumin $(r=-0.458$ and $p=0.000)$. (Table 2$)$. There was no positive or negative correlation with CRP blood parameter APACHE-II and SOFA score ( $p=0.452, p=0.640$ ) (Table 3). In our study, we divided into two groups as survivors according to 28-day mortality. Threshold values of blood parameters were determined by roc analysis in terms of mortality. procalcitonin $37.7 \mathrm{ng} / \mathrm{mL}$ AUC: 0.616 (0.447- 0.785), Albumin 2.06 g/dL AUC: 0.829 (0.717-0.941), procalcitonin/albumin 18.5 AUC: 0.645 (0.479- 0.811), Lactate cut of value $1.5 \mathrm{mEq} / \mathrm{L}$ AUC: 0.693 (0.525- 0.861), CRP $39 \mathrm{mg} / \mathrm{L}$ AUC: 0.648 (0.483-0.812) (Figure 3). procalcitonin, CRP, lactate, procalcitonin/albumin in terms of mortality in the evaluation of the area under the curve $>0.5$ is considered to be significant.

\section{Discussion}

Prolongation of the life period and improved healthcare services caused a trend of increase in critical patients recently. Critical patients who develop one or multiple organ failure have cardiac, respiratory or neurological diseases and need supplementary treatments such as intubation, mechanical ventilation and positive inotropic agents (16). Sensitive and specific scoring systems and laboratory tests are required to guide monitoring of treatment response in critical patients. In this study, blood parameters with acute phase reactants were evaluated in terms of determining mortality, and these parameters were compared in terms of clinical progress and correlation in relation to prognostic scores including APACHE-II and SOFA.

The relationship between low serum albumin levels and severe disease and poor prognosis has been shown for many years in different patient groups with many studies (17). In a study, they reported that the mortality rate was $24 \%$ in hospitalized patients with serum albumin concentration lower than $3.4 \mathrm{mg} / \mathrm{dL}$ and $62 \%$ in patients with lower than $2 \mathrm{mg} / \mathrm{dL}$ (18). As a result of our study, albumin has similar results with other studies; SOFA and APACHE-II scores were negatively correlated with moderate level. Albumin value was an important factor in mortality. Some recent studies detected increased lactate concentration with higher mortality in different patient groups (19). Yılmaz et al. (20) conducted a retrospective study on trauma patients and detected a significant association between higher lactate level and mortality.

A previous studies on sepsis emphasized that the value of lactate was important in terms of mortality $(21,22)$. In the present study, the association between lactate level and SOFA scoring was moderately significant and it was shown as an important factor on mortality. In addition, the lactate cut-off point was found to be $1.5 \mathrm{mEq} / \mathrm{L}$ according to Roc analysis and it was found to 


\begin{tabular}{|c|c|c|c|c|c|c|c|}
\hline $\begin{array}{l}\text { Demographic characteristics - } \\
\text { independent variables (IVs) }\end{array}$ & Name of characteristics & Label & Number & Percent (\%) & Mean & SD & Scale \\
\hline \multirow{3}{*}{ Gender } & Female $(0)$ & FEML & 26 & 42.6 & \multirow{3}{*}{0.57} & \multirow{3}{*}{0.499} & \multirow{3}{*}{$0-1$} \\
\hline & Male (1) & MALE & 35 & 57.4 & & & \\
\hline & Total & - & 61 & 100 & & & \\
\hline Age & Total & AGE & 61 & 100 & 65.77 & 18.51 & $19-91$ \\
\hline \multirow{10}{*}{ Additional disease } & Diabetes Melitus (1) & DM & 19 & 31.1 & - & - & - \\
\hline & Hipertansiyon (2) & $\mathrm{HT}$ & 25 & 41.0 & - & - & - \\
\hline & Cerebrovasculer disease (3) & CVD & 8 & 13.1 & - & - & - \\
\hline & Coronary artery disease (4) & CAD & 15 & 24.6 & - & - & - \\
\hline & Chronic kidney disease (5) & CKD & 9 & 14.8 & - & - & - \\
\hline & Alzheimer (6) & ALZH & 3 & 4.9 & - & - & - \\
\hline & Cancer (7) & CA & 11 & 18.0 & - & - & - \\
\hline & $\begin{array}{l}\text { Chronic obstructive } \\
\text { pulmonary disease (8) }\end{array}$ & COPD & 18 & 29.5 & - & - & - \\
\hline & Other (9) & Other & 16 & 26.2 & - & - & - \\
\hline & Total & - & - & - & - & 0.321 & $1-9$ \\
\hline \multirow{14}{*}{ Diagnosis of hospitalisation } & Pneumonia (1) & PNM & 28 & 45.9 & - & - & - \\
\hline & Sepsis (2) & SPS & 9 & 14.8 & - & - & - \\
\hline & Hypoxic respiratory failure (3) & HSY & 16 & 26.2 & - & - & - \\
\hline & Akut kidney disease (4) & $\mathrm{ABY}$ & 12 & 19.7 & - & - & - \\
\hline & Peritonit (5) & PRT & 2 & 3.3 & - & - & - \\
\hline & Electrolyte disturbance (6) & $\mathrm{EB}$ & 6 & 9.8 & - & - & - \\
\hline & Diabetic ketoacidosis (7) & DKA & 3 & 4.9 & - & - & - \\
\hline & Cerebrovascüler disease (8) & SVO & 3 & 4.9 & - & - & - \\
\hline & $\begin{array}{l}\text { Gastrointetinal } \\
\text { hospitalisation (9) }\end{array}$ & GISH & 6 & 9.8 & - & - & - \\
\hline & Trauma (10) & TRV & 4 & 6.6 & - & - & - \\
\hline & Pulmonary embolism (11) & $\mathrm{PE}$ & 3 & 4.9 & - & - & - \\
\hline & Subaracnoid K (12) & SAK & 1 & 1.6 & - & - & - \\
\hline & Other (13) & OTHER & 8 & 13.1 & - & - & - \\
\hline & Total & - & 61 & 100 & - & - & $1-13$ \\
\hline \multirow{6}{*}{ Glaskow Coma score } & $15(1)$ & GCS1 & 26 & 42.6 & - & - & - \\
\hline & $13-14(2)$ & GCS2 & 16 & 26.2 & - & - & - \\
\hline & $12-10(3)$ & GCS3 & 11 & 18.0 & - & & \\
\hline & $9-6(4)$ & GCS4 & 6 & 9.8 & - & - & - \\
\hline & $<6(5)$ & GCS5 & 2 & 3.3 & - & - & - \\
\hline & Total & - & 61 & 100 & 2.04 & 1.14 & $1-5$ \\
\hline \multirow{5}{*}{ Intensive care duration } & $1-3(1)$ & - & 21 & 34.4 & - & - & - \\
\hline & $4-7(2)$ & - & 22 & 36.1 & - & - & - \\
\hline & $8-14(3)$ & - & 13 & 21.3 & - & - & - \\
\hline & $>14(4)$ & - & 5 & 8.2 & - & - & - \\
\hline & Total & - & 61 & 100 & 2.03 & 0.94 & $1-4$ \\
\hline \multirow{5}{*}{ Clinical navigation } & Exitus (1) & - & 15 & 24.6 & - & - & - \\
\hline & Healthy discharged (2) & - & 17 & 27.9 & - & - & - \\
\hline & Transfer to the service (3) & - & 9 & 14.8 & - & - & - \\
\hline & Intensive care referral (4) & - & 20 & 32.8 & - & - & - \\
\hline & Total & - & 61 & 100 & - & 1.190 & $1-4$ \\
\hline
\end{tabular}




\begin{tabular}{|c|c|c|c|c|c|}
\hline \multicolumn{3}{|l|}{ Correlations } & \multirow{2}{*}{$\begin{array}{l}\text { Procalcitonin } \\
0.331 \\
\end{array}$} & \multirow{2}{*}{$\begin{array}{l}\text { Albumin } \\
-0.465\end{array}$} & \multirow{2}{*}{$\begin{array}{l}\text { Procalcitonin/ } \\
\text { albumin } \\
0.362 \\
\end{array}$} \\
\hline \multirow{6}{*}{ Spearman's rho } & \multirow{3}{*}{ SOFA } & Cor. Coef. & & & \\
\hline & & Sig. & 0.009 & 0.000 & 0.004 \\
\hline & & $\mathrm{N}$ & 61 & 61 & 61 \\
\hline & \multirow{3}{*}{ APACHE II } & Cor. Coef. & 0.254 & -0.469 & 0.288 \\
\hline & & Sig. & 0.048 & 0.000 & 0.025 \\
\hline & & $N$ & 61 & 61 & 61 \\
\hline
\end{tabular}

Table 3. correlation between CRP, procalcitonin, albumin, lactate and procalcitonin/albumin among laboratory parameters and APACHE-II and SOFA

\begin{tabular}{|l|l|l|l|l|}
\hline \multicolumn{2}{|l|}{ Correlations } & Lactat & CRP \\
\hline \multirow{4}{*}{ SOFA } & Cor. Coef. & 0.353 & 0.098 \\
\cline { 3 - 5 } & Sig. & 0.005 & 0.640 \\
\cline { 3 - 5 } & N & 61 & 61 \\
\cline { 3 - 5 } & \multirow{4}{*}{ APACHE II } & Cor. Coef. & 0.316 & 0.061 \\
\cline { 3 - 5 } & \multirow{3}{*}{ Sig. } & 0.013 & 0.452 \\
\cline { 3 - 5 } & \multirow{2}{*}{ N } & 61 & 61 \\
\hline
\end{tabular}

APACHE II: Acute Physiology And Chronic Health Evaluation II, SOFA: The sequential organ failure assessment score, Cor: Correlation, Coef: Coefficient, Sig: Significant, CRP: C-reactive protein, N: Number

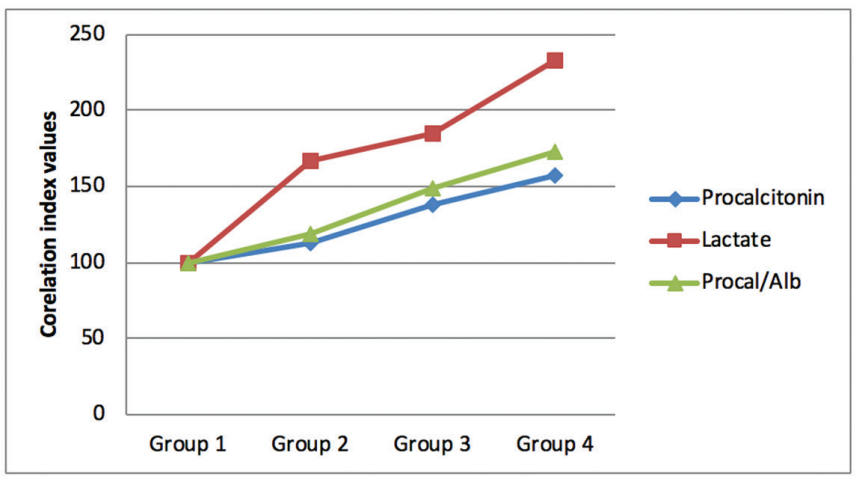

Figure 1. Correlation values of blood parameters according to APACHE II score groups

APACHE II: Acute Physiology And Chronic Health Evaluation II, Alb: Albumin

be an important factor on mortality. There is not any positive or negative linearity between CRP levels and both APACHE-II and SOFA scores. A recent study did not show any effect of CRP acute phase reactant on mortality in trauma patients followed in intensive care unit (20). Although CRP did not correlate with SOFA and APACHE-II, CRP cut-off value $>39 \mathrm{mg} / \mathrm{dL}$ was found to be significant in terms of mortality. Recent studies addressed

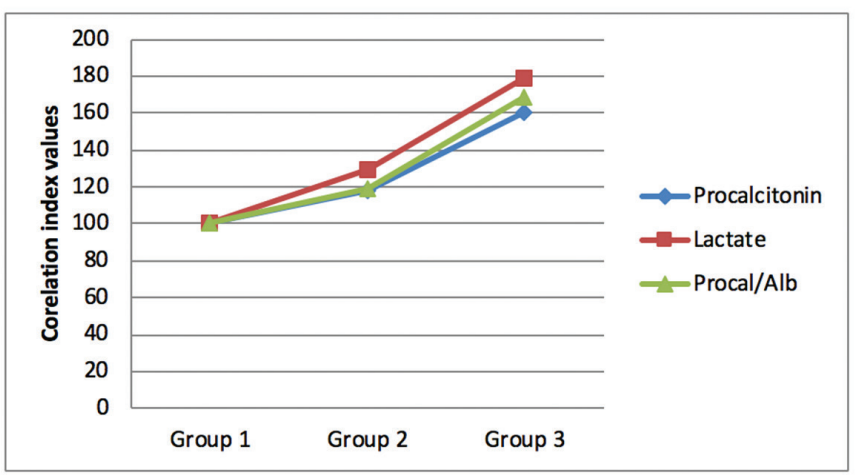

Figure 2. Correlation values of blood parameters according to SOFA score groups

SOFA: The sequential organ failure assessment score, Alb: Albumin



Figure 3. Mortality of blood parameters according to ROC analysis (Procalcitonin 37.7 ng/mL AUC:0.616 (0.447-0.785), Albumin 2.06 g/dLAUC: 0.829 (0.717-0.941), procalcitonin/albumin 18.5, AUC: 0.645 (0.479-0.811), lactate cut off value $1.5 \mathrm{mEq} / \mathrm{mL}$ AUC: 0.693 (0.525- 0.861), CRP: 39 mg/L AUC: 0.648 (0.483-0.812)

ROC: Receiver operating characteristic, AUC: Area under curve, CRP: C-reactive protein

that procalcitonin increase above $2 \mathrm{ng} / \mathrm{mL}$ in clinical conditions such as severe infection, sepsis and multiple organ failure 
syndrome, and it is an important indicator for early diagnosis $(23,24)$. Procalcitonin is a specific and sensitive marker for severe bacterial infections which may show developing inflammatory response earlier $(25,26)$. Similar results were obtained in the present study. It was considered that procalcitonin presents a positive correlation with APACHE-II and SOFA scores; and it is a useful laboratory parameter for monitoring and treatment. High procalcitonin levels are associated with the severity of the infection and have been shown to be used in the follow-up of patients with severe infections, sepsis, and multiple organ failure syndrome (25). In a study, procalcitonin and albumin were important markers of infection in elderly individuals (27). In our study, procalcitonin and procalcitonin/albumin ratio were found to have a low positive correlation with prognostic scores and were found to be helpful parameters during the clinical course.

In the ROC analysis, the AUC value of the blood parameters is above 0.5 and the AUC value of the albumin is high. This shows us the importance of the albumin cut-off value in terms of mortality below $2.06 \mathrm{~g} / \mathrm{dL}$. In the study of Yap et al. (28), Similar results were obtained with our study and it was emphasised that albumin may be associated with mortality.

\section{Study Limitations}

This study was performed in the intensive care unit and the patient population was limited.

\section{Conclusion}

Considering the importance of critical patients on mortality since admission to the emergency department, procalcitonin, lactate albumin and procalcitonin/albumin were thought to be prognostic markers according to the cut-off points. In addition, they were found to be beneficial in clinical follow-up as they correlated with the scores of APACHE-II and SOFA scoring systems used in intensive care units.

\section{Ethics}

Ethics Committee Approval: Ethical approval for this study was obtained from the Ethics Committee of Kanuni Sultan Süleyman Training and Research Hospital with the protocol numbered 2019/07/175.

Informed Consent: Retrospective study.

Peer-review: Externally and internally peer-reviewed.

\section{Authorship Contributions}

Surgical and Medical Practices: D.A., R.G., Concept: B.C., R.Y., T.Ö.D., Design: D.A., B.C., R.G., Data Collection or Processing: D.A., B.B., H.K., T.Ö.D., R.G., Analysis or Interpretation: B.C., R.Y., R.G., Literature Search: D.A., B.B., Writing: D.A., T.Ö.D.
Conflict of Interest: No conflict of interest was declared by the authors.

Financial Disclosure: The authors declared that this study received no financial support.

\section{References}

1. Waydhays C. Equipment review. Intrahospital transport of critically ill patients. Crit Care Med. 1999;5:83-9.

2. Robertson LC, Al-Haddad M. Recognizing the critically ill patient. Anaesthesia \& Intensive Care Medicine. 2013;14:11-4

3. Guidelines for intensive care unit admission, discharge, and triage. Task Force of the American College of Critical Care Medicine, Society of Critical Care Medicine. Crit Care Med. 1999;27:633-8.

4. Lee CK, TH Rainer TH. Application of APACHE II in the assessment, classification of severity and predictive ability of Chinese patients presenting to an emergency department resuscitation room. Hong Kong Journal of Emergency Medicine. 2002;9:188-94.

5. Ferreira FL, Bota DP, Bross A, et al. Serial Evaluation of the SOFA Score to Predict Outcome in Critically III Patients. JAMA. 2001;286:1754-8.

6. Castelli GP, Pognani C, Cita M, Stuani A, Sgarbi L, Paladini R. Procalcitonin, C-reactive protein, white blood cells and SOFA score in ICU: diagnosis and monitoring of sepsis. Minerva Anestesiol. 2006;72:69-80.

7. Köse B, Özcan N, Kaymak C, Bașar H, Kotanoğlu M, Özcan A, et al. Assessment of Scoring Systems, Procalcitonin Levels and Blood Gas Parameters Used in Follow Up of Septic and Non-Septic Patients. Turkiye Klinikleri J Anest Reanim. 2013;11:137-42.

8. Smith RP, Lipworth BJ. C-reactive protein in simple community acquired pneumonia. Chest. 1995;107:1028-31.

9. Hamm CW, Nef HM, Rolf A, Möllmann H. Calcium and C-reactive protein. J Am Coll Cardiol. 2011:57:465-7.

10. Whicher J, Bienvenu J, Monneret G. Procalcitonin as an acute phase marker. Ann Clin Biochem .2001;38:483-93.

11. Müller B, White JC, Nylén ES, Snider RH, Becker KL, Habener JF. Ubiquitous expression of the calcitonin-I gene in multiple tissues in response to sepsis. J Clin Endocrinol Metab. 2001;86:396-404.

12. Ouellet JF, Roberts DJ, Tiruta C, Kirkpatrick AW, Mercado M, Trottier V, et al Admission base deficit and lactate levels in Canadian patients with blunt trauma: Are they useful markers of mortality? J Trauma Acute Care Surg. 2012;72:1532-5

13. Boldt J, Suttner S. Plasma subsitutes. Minerva Anestesiol. 2005;7:741-58

14. Kaysen G. Biological Basis of Hypoalbuminemia J Am Soc Nephrol. 1998; 9:2368-76.

15. Knaus WA, Draper EA, Wagner DP. APACHE II: A severity of disease classification system. Crit Care Med. 1985;13:818-29.

16. Cander B, Dundar ZD, Gul M, Girisgin G. Prognostic value of serum zinc levels in critically ill patients. Journal of Critical Care. 2011;26:42-6.

17. Törer B. Prematüre Bebeklerde Hipoalbumineminin Yenidoğan Dönemi Sorunları ve Mortalite Üzerine Etkisi (Tez), Ankara: Bașkent Üniversitesi Tıp Fakültesi. 2010

18. Reinhardt GF, Myscofski JW, Wilkens DB, Dobrin PB, Mangan JE Jr, Stannard RT. Incidence and mortality of hypoalbuminemic patients in hospitalized veterans. JPEN J Parenter Enteral Nutr. 1980;4:357-9.

19. Luft D, Deichsel G, Schmülling RM, Stein W, Eggstein M. Definition of clinically relevant lactic acidosis in patients with internal diseases. Am J Clin Pathol. 1983;80:484-9.

20. Yılmaz E, Bor C, Uyar M, Demirağ K, Çankayalı I. The Effect of Lactate, Albumin, C-reactive Protein, PaO2/FiO2 and Glucose Levels of Trauma 
Patients at the Time of Administration to Intensive Care Unit on Mortality. Journal of the Turkish Society of Intensive Care. 2014;12: 82-5.

21. Yang MLC, Graham CA, Rainer TH. Outcome after implementation of sepsis guideline in the emergency department of a university hospital in Hong Kong. Hong Kong J Emerg Med. 2015;22:163-71.

22. Baumann, BM, Greenwood JC, Lewis K, Nuckton TJ, Darger B, Shofer FS, et al. Combining qSOFA criteria with initial lactate levels: Improved screening of septic patients for critical illness. Am J Emerg Med. 2019 Jul 4;50735675730450-4. doi: 10.1016/j.ajem.2019.07.003. [Epub ahead of print]

23. Karzai W, Oberhoffer M, Meier-Hellmann A, Reinhart K. Procalcitonin a new indicator of the systemic response to severe infection. Infection. 1997;25:32934.

24. Steinhach G, Grünert A. Procalcitonin a new indicator for bacterial infection. Exp Clin Endocrinol Diabetes. 1998;106:164-7.
25. Carrol ED, Thomson AP, Hart CA. Procalcitonin as a marker of sepsis. Int Antimicrob Agents. 2002;20:1-9.

26. Rebello A, Thabah MM, Dutta TK, Bobby Z, Harrish BN, Mehalingam V. Procalcitonin levels in sepsis and its association with clinical outcome in southern India. Tropical Doctor. 2017;47:331-6.

27. Higashikawa T, Okuro M, Ishigami K, Mae K, Sangen R, Mizuno T, et al. Procalcitonin and albumin as prognostic biomarkers in elderly patients with a risk of bacterial infection. Journal of International Medical Research. 2018:46;2606-14.

28. Yap FHY, Joynt GM, Buckley TA, Wong ELY. Association of Serum Albumin Concentration and Mortality Risk in Critically III Patients. Anaesth Intensive Care. 2002;30:202-7. 